\title{
Mechanochemical synthesis of hyper-crosslinked polymers: influences on their pore structure and adsorption behaviour for organic vapors
}

\author{
Sven Grätz ${ }^{* 1}$, Sebastian Zink ${ }^{2}$, Hanna Kraffczyk ${ }^{3}$, Marcus Rose ${ }^{3}$ and Lars Borchardt ${ }^{* 1}$
}

\author{
Full Research Paper \\ Address: \\ ${ }^{1}$ Anorganische Chemie, Ruhr-Universität Bochum, Universitätsstraße \\ 150, 44801 Bochum, Germany, ${ }^{2}$ Institute of Inorganic Chemistry, \\ Technische Universität Dresden, Dresden, Germany and ${ }^{3}$ Fachgebiet \\ Technische Chemie II, Technische Universität Darmstadt, Darmstadt, \\ Germany \\ Email: \\ Sven Grätz - sven.graetz@rub.de; Lars Borchardt ${ }^{*}$ - \\ lars.borchardt@ruhr-uni-bochum.de \\ * Corresponding author \\ Keywords: \\ hyper-crosslinked polymers; mechanochemistry; microporous; \\ solvent-free; vapor sorption \\ Beilstein J. Org. Chem. 2019, 15, 1154-1161. \\ doi:10.3762/bjoc. 15.112 \\ Received: 08 February 2019 \\ Accepted: 16 May 2019 \\ Published: 24 May 2019 \\ This article is part of the thematic issue "Mechanochemistry II". \\ Guest Editor: J. G. Hernández \\ (C) 2019 Grätz et al.; licensee Beilstein-Institut. \\ License and terms: see end of document.
}

Open Access

\begin{abstract}
This study elucidates a mechanochemical polymerization reaction towards a hyper-crosslinked polymer as an alternative to conventional solvent-based procedures. The swift and solvent-free Friedel-Crafts alkylation reaction yields a porous polymer with surface areas of up to $1720 \mathrm{~m}^{2} \mathrm{~g}^{-1}$ and pore volumes of up to $1.55 \mathrm{~cm}^{3} \mathrm{~g}^{-1}$. The application of LAG (liquid-assisted grinding) revealed a profound impact of the liquid's boiling point on the textural properties of the obtained polymer materials. Finally, the materials are characterized by vapour sorption experiments with benzene and cyclohexane.
\end{abstract}

\section{Introduction}

The widespread use of microporous materials in areas like gas storage, gas separation, and catalysis has led to the development of a wide variety of these materials [1]. While inorganic materials such as activated carbons, porous metal oxides, and zeolites have been investigated for decades, hybrid materials such as metal-organic frameworks [2], purely organic frameworks such as crystalline covalent-organic frameworks [3], and amorphous POPs (porous organic polymers) [4] are currently in the focus of research. The modular bottom-up building concept of these organic materials allows for tailoring of materials properties towards desired applications. POPs synthesis can be achieved by a huge variety of reactions ranging from FriedelCrafts alkylations [5], cross-coupling reactions [6] and cyclotrimerizations [7], to amine-based chemistry with Schiff base reactions [8], imidisation, and amidisation reactions [9]. In the recent past, a strong focus was also set on the development of HCPs (hyper-crosslinked polymers) [10]. Typically, they are formed by a non-directed aromatic substitution (Friedel-Crafts alkylation) either by intramolecular functional groups or using external crosslinkers. These reactions yield very high crosslinking degrees in amorphous framework structures and hence, enable highest specific BET (Brunauer-Emmett-Teller) sur- 
face areas of up to $2000 \mathrm{~m}^{2} \mathrm{~g}^{-1}$ for fully amorphous materials [10]. Typically, chloromethyl or methoxy groups are used to crosslink aromatic building blocks by using stoichiometric or even excess amounts of $\mathrm{FeCl}_{3}$ as catalyst. Recently, also a metal-free Brønsted acid-catalyzed reaction using trifluoromethanesulfonic acid or sulfuric acid was developed yielding basically the same polymer but avoiding residual traces of the metal [11]. These synthetic pathways often afford a gel from the dissolved monomers upon the crosslinking that is subsequently washed and dried with a significant volume loss. Still, a high amount of permanent micro- and mesoporosity is retained. This gel formation is kinetically fast and thus, hard to control. Hence, an initial intensive mixing is crucial to achieve a homogeneous material. Also, due to the shrinkage upon the drying step, a high flexibility of the framework remains as an intrinsic property. Thus, the pore volume and specific surface area that is permanently accessible can be increased by a severe swelling behaviour especially during the adsorption and combined with absorption of organic vapors or liquids. The swelling is fully reversible, thus, rendering these materials interesting adsorbents with a dynamic adsorption behaviour.

However, the low solubility of POPs is a main challenge in their synthesis protocols. Solution-based procedures, for example, suffer from almost instant precipitation and thus only produce materials with a low degree of polymerization [10]. In the recent past it has been shown that for reactions, where the solu- bility of the reactants or products is an issue, mechanochemistry - a field which is currently gaining momentum - can be a promising workaround [12-20]. It has already been established as a versatile tool for the synthesis of several porous materials [21-29] and polymers [30-42].

In this contribution, we employed mechanochemistry for a protocol for the solvent-free crosslinking of HCP (Figure 1). This is supported by an investigation of chemical and milling parameters on the yield and surface area of the resulting polymer.

\section{Results and Discussion Polymer synthesis and characterization}

In our standard procedure HCP was synthesised via a mechanochemical reaction. This was accomplished by transferring $\mathrm{BCMCP}$ (4,4'-bis(chloromethyl)-1,1'-biphenyl) and six equiv of anhydrous iron(III) chloride as mediator into a $45 \mathrm{~mL}$ zirconium oxide milling vessel filled with 22 balls $(10 \mathrm{~mm}$, $3.19 \pm 0.05 \mathrm{~g}$ ) of the same material (Figure 1). Consequently, the vessel was transferred into a (Pulverisette 7) premium line (Fritsch $\mathrm{GmbH}$ ) and milled at $500 \mathrm{rpm}$ for 35 minutes. After the synthesis, the resulting polymers were washed with $200 \mathrm{~mL}$ of water and $100 \mathrm{~mL}$ of ethanol and dried at $80{ }^{\circ} \mathrm{C}$ for 12 hours to yield a beige powder (denoted NG-HCP (neat grinding-HCP)). For more details on the experimental procedure see Supporting Information File 1.
A

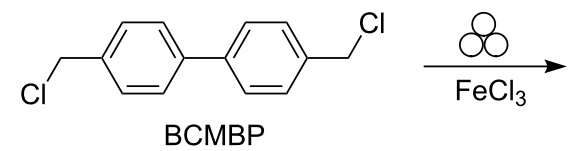

B

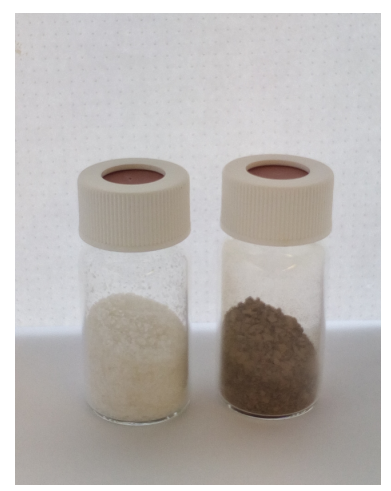<smiles>CCc1ccc(-c2ccc(Cc3cc(CC)c(Cc4ccc(-c5ccc(CC)cc5)cc4CC)cc3-c3ccc(CC)cc3)cc2)cc1</smiles>

HCP 
After validating the complete insolubility in common organic solvents (like DCM, ethanol, DMF, chlorofrom, $n$-hexane), the nature of the polymer was investigated via IR spectroscopy. In the past the $\mathrm{C}-\mathrm{Cl}$ vibration at $680 \mathrm{~cm}^{-1}$ has commonly been used to establish whether the desired crosslinking via the chloromethylene groups has occurred [43]. While this vibration is dominant in the monomer, it has completely vanished in the IR (infrared) spectrum of the polymer (Figure 2A). Moreover, several other changes in the spectrum are observed for the polymer as well. Especially in the region of the $\mathrm{C}-\mathrm{H}_{\text {oop }}$ the vibration is shifted to $885 \mathrm{~cm}^{-1}$ hinting towards one isolated hydrogen atom which further confirms the cross-linking between the monomers. The creation of these aliphatic connections is also evident based on the appearance of vibrations around $1400 \mathrm{~cm}^{-1}$ [43]. As reported in the literature for the solvent-based protocol the polymer obtained through this mechanochemical route is also amorphous as visualized by the XRD (X-ray diffraction) patters recorded (Supporting Information File 1, Figure S1) [43].

Furthermore, NG-HCP similarly shows a high thermal stability with a decomposition onset in air (Supporting Information File 1, Figure S2) at around $310^{\circ} \mathrm{C}$. The residual mass of only $1.9 \%$ confirms the absence of both, excessive abrasion and oxidant inside the polymer. This is further supported by the SEM-EDX (scattering electron microscope, energy-dispersive $\mathrm{X}$-ray spectroscopy) measurements showing both $\mathrm{Cl}$ and $\mathrm{Fe}$ contents below the limit of detection (Supporting Information File 1, Figure S3). Scattering electron microscopy also revealed an agglomerated morphology of the samples (Supporting Information File 1, Figure S4).

After successfully establishing the occurrence of the crosslinking reaction and investigating the physical and thermal properties of the polymer, the textural properties of the NG-HCP were evaluated utilizing nitrogen physisorption. While the first tries directly yielded a microporous polymer $\left(\mathrm{SSA}_{\mathrm{BET}}=850 \mathrm{~m}^{2} \mathrm{~g}^{-1}, V_{\mathrm{p}}=0.60 \mathrm{~cm}^{-3} \mathrm{~g}^{-1}\right.$, Figure $2 \mathrm{~B}$ in Supporting Information File 1), the SSA (specific surface area) achieved with the classical solvent-based approach (Sol-HCP, $\mathrm{SSA}_{\mathrm{BET}}=1450 \mathrm{~m}^{2} \mathrm{~g}^{-1}, V_{\mathrm{p}}=1.55 \mathrm{~cm}^{-3} \mathrm{~g}^{-1}$ ) [11] could not be reached. In order to improve this property, we varied the mechanochemical reaction parameters such as milling time and amount of oxidant in a systematic (DOE) design of experiments approach (see Supporting Information File 1 for details). While changes in these parameters translated to variations in the specific surface, it was not possible to achieve surface areas as high as the solution-based method solely by adapting the aforementioned parameters. The most influential parameter was determined to be the rotational speed of the ball mill. At $200 \mathrm{rpm}$ this led to a low SSA $\left(7-66 \mathrm{~m}^{2} \mathrm{~g}^{-1}\right)$ due to an incomplete polymerization, while $800 \mathrm{rpm}$ resulted in a medium SSA $\left(600-820 \mathrm{~m}^{2} \mathrm{~g}^{-1}\right)$. This observation is hinting towards a partial degradation of the porosity by the high energy input and the resulting thermal and frictional stress of the material and reinforces our prior findings regarding the synthesis of porous polymers inside a ball mill $[30,35]$.

\section{Development of vessel pressure during the reaction}

In an attempt to track and understand the kinetics of the mechanochemical polymerisation further, we employed the so-called GTM-system (gas pressure and temperature measurement system), which allows us to measure the temperature and pressure inside the milling vessel during the milling process. While a release of gas was observed when opening the vessel, the GTM measurement revealed that even small amounts of the monomer (3.2 mmol) lead to a swift and rise in vessel pressure
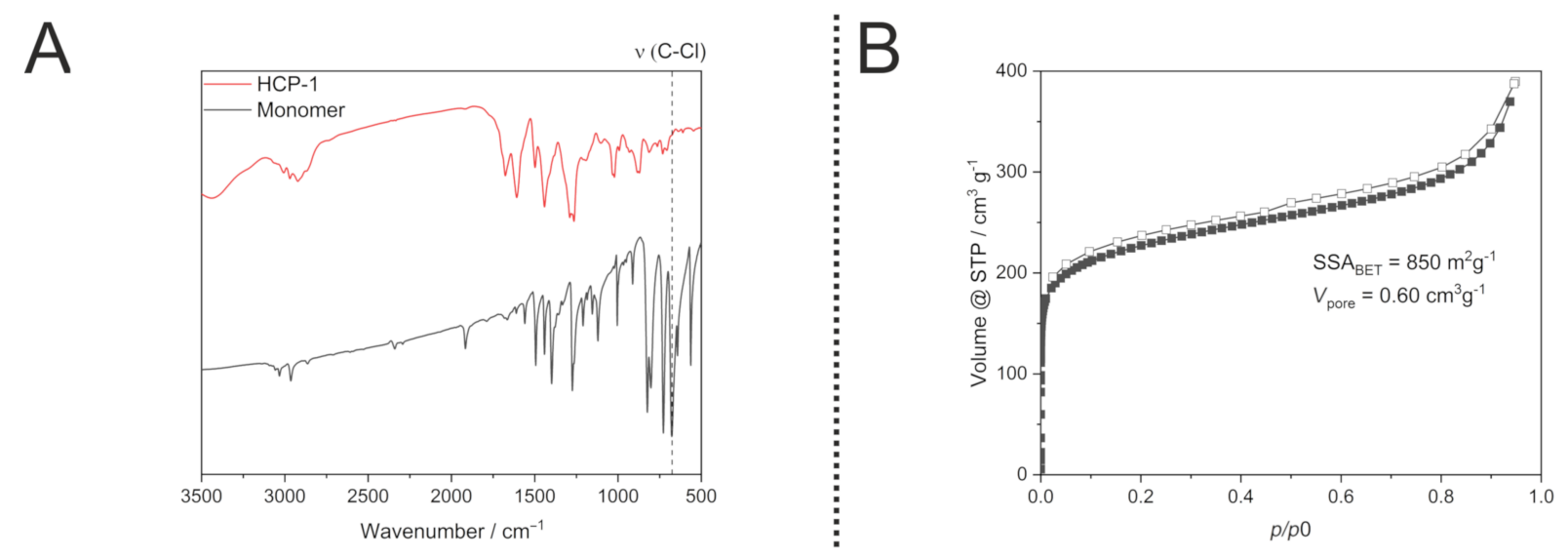

Figure 2: A: IR spectra of the monomer BCMBP and NG-HCP showing a decrease of the $\mathrm{C}-\mathrm{Cl}$ vibration after the reaction. B: Low pressure nitrogen isotherm of NG-HCP demonstrating the textural properties. 
exceeding 12 bar (Figure $3 \mathrm{~A}$ ) caused by the released $\mathrm{HCl}$ in the course of the reaction. This behaviour can be handled under laboratory conditions but is hampering the potential scale up of the process. In the recent past we have already observed this behaviour for other reactions including iron(III) chloride [42,44], and thus we have proposed several countermeasures which greatly reduce the vessel pressure during such reactions. For this polymerization reaction we decided to add small quantities of liquid to dissolve the gas, as the method of choice. At first $1 \mathrm{~mL}$ of ethanol was added to the reaction mixture prior to the milling. This measure resulted in a decrease of vessel pressure of the whole course of the reaction. With diethyl ether instead of ethanol the pressure could be reduced even more limiting the maximum pressure to 0.42 bar (Figure 3B).

\section{Influence of the LAG additive}

Interestingly, besides decreasing the vessel pressure, this $1 \mathrm{~mL}$ of solvent also affected the textural properties of the polymer positively. Consequently, we went on to investigate the influence of the LAG reagent by changing the nature of the latter. The surface area of the polymer was increased up to $1720 \mathrm{~m}^{2} \mathrm{~g}^{-1}$ when utilizing diethyl ether (Table 1, entry 7), therefore almost doubled, while the pore volume is also growing. In the pore size distribution one can observe a shift from $0.75 \mathrm{~nm}$ micropores for NG-HCP to a mixture of 0.67 and $0.97 \mathrm{~nm}$ micropores for LAG-HCP (Figures S5 and S6 in Supporting Information File 1). In addition, the amount of mesopores in the sample is also slightly higher for the LAG sample. The solubility of the released gas inside the liquid seems to play a minor role since good results can be obtained with either dichloromethane (poor $\mathrm{HCl}$ solvent) as well as diethyl ether (good $\mathrm{HCl}$ solvent). On the other hand, the boiling point of the added liquid seems to be an important parameter for the surface area of the synthesised polymer, with lower boiling point solvents resulting in higher surface areas than their high boiling point counterparts (Figure 4). This might indicate that the additive acts as a porogene in the synthesis of the polymer, with the vapour being a more effective than the liquid. However, to test this hypothesis a profound control over the vessel temperature is necessary which cannot be achieved with our current experimental setup but is generally possible [45]. Nevertheless, the GTM systems showed that macroscopic temperatures exceeding the boiling points of dichloromethane and diethyl ether have been reached in the course of the experiments. Since the best results were obtained for diethyl ether (Table 1, entry 7) this sample has been denoted LAG-HCP and was investigated further.

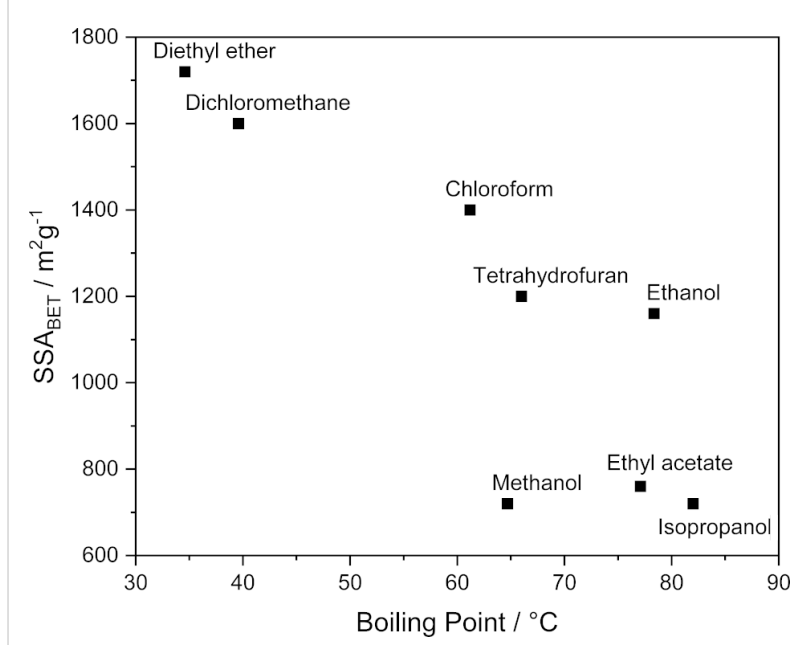

Figure 4: The correlation between the liquids' boiling point and the SSA of the polymer. In general, a lower boiling point of the LAG additive leads to a higher SSA.

\section{Vapor physisorption}

Since HCP is highly hydrophobic (proven before by water vapor physisorption [11]), the adsorption of non-polar adsorp-
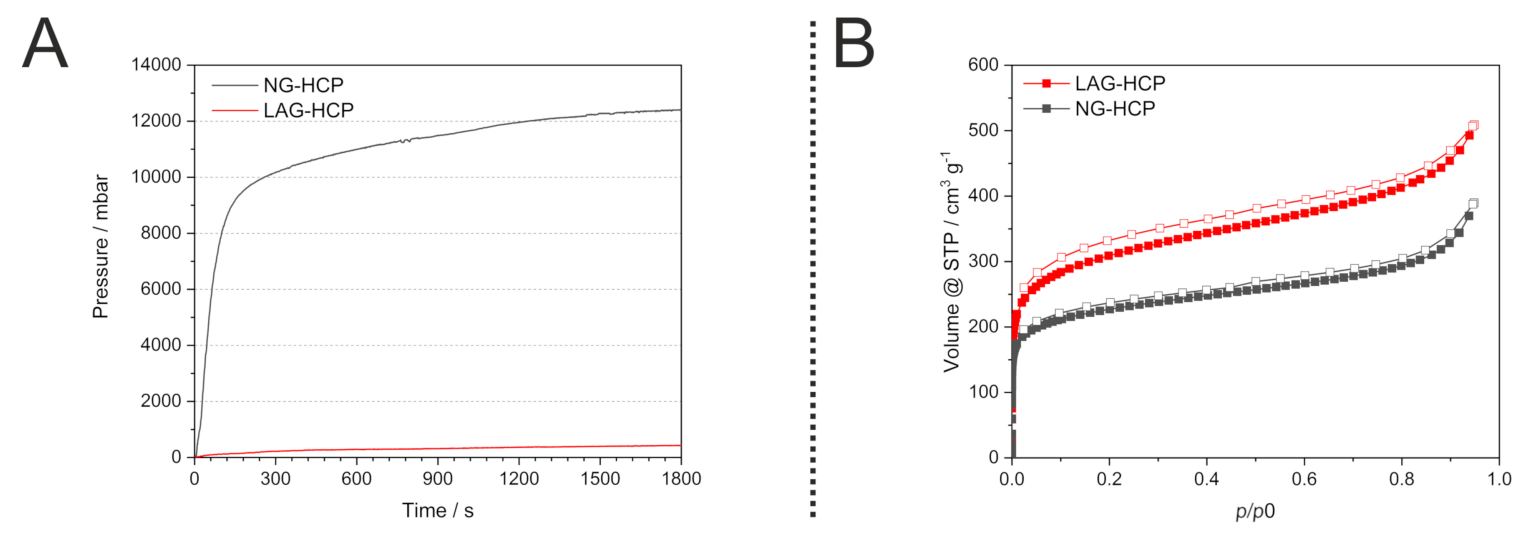

Figure 3: A: Evolution of pressure in the course of the reaction measured by the GTM system. The addition of $1 \mathrm{~mL}$ of Et $\mathrm{t}_{2} \mathrm{O}$ is sufficient to drastically reduce the released pressure. B: Comparison of the low pressure nitrogen isotherm of NG-HCP and LAG-HCP. 
Table 1: Influence of the LAG additive on the textural properties of HCPa.

\begin{tabular}{llll} 
Entry & Liquid additive & $\mathrm{SSA}_{\mathrm{BET}}{ }^{\mathrm{b}}\left[\mathrm{m}^{2} \mathrm{~g}^{-1}\right]$ & $V_{\mathrm{Pore}^{\mathrm{C}}\left[\mathrm{cm}^{3} \mathrm{~g}^{-1}\right]}$ \\
\hline 1 & - & 850 & 0.60 \\
2 & ethanol & 1160 & 0.98 \\
3 & methanol & 720 & 0.61 \\
4 & chloroform & 1400 & 0.91 \\
5 & dichloromethane & 1550 & 1.12 \\
6 & isopropanol & 720 & 0.52 \\
$7^{\mathrm{d}}$ & diethyl ether & 1720 & 1.06 \\
8 & ethyl acetate & 760 & 0.51 \\
9 & tetrahydrofuran & 1200 & 1.00 \\
$10^{\mathrm{e}}$ & - & 1450 & 1.55 \\
\hline
\end{tabular}

aAll samples were synthesised under the following conditions: $35 \mathrm{~min}, 500 \mathrm{rpm}, 6$ equiv $\mathrm{FeCl}_{3}, 22$ balls á $10 \mathrm{~mm}, 1 \mathrm{~mL}$ of liquid additive; baccording to the Brunauer-Emmett-Teller theory, utilizing the Rouquerol method; ' determined at $p / p_{0}=0.99$; ${ }^{\text {in }}$ the following denoted as LAG-HCP; ${ }^{\text {solution- }}$ based references, Sol-HCP.

tives in the gas and liquid phase is strongly favored. Recently, the advantage of HCP over various commonly available adsorbents in the selective liquid phase adsorption of least polar component in aqueous solution has been demonstrated [46,47]. Herein, the adsorption properties for the organic vapors benzene and cyclohexane are reported and the differences between LAG- HCP and the solvent-based reference material Sol-HCP are demonstrated.

First of all, is has to be noted that Sol-HCP (reference according to Schute et al. [11]) showed a specific BET surface area of $1450 \mathrm{~m}^{2} \mathrm{~g}^{-1}$, hence, slightly below the LAG-HCP with a SSA of $1720 \mathrm{~m}^{2} \mathrm{~g}^{-1}$. Still, it showed a higher total pore volume of $1.55 \mathrm{~cm}^{3} \mathrm{~g}^{-1}$ due to the higher ratio of mesopores and hence, a lower share of micropore volume $\left(0.24 \mathrm{~cm}^{3} \mathrm{~g}^{-1}\right)$. Also, the pore size distribution proved to be rather broad (as reported before) compared to the mechanochemically synthesised material. For both materials the overall uptake of benzene is higher than for previously reported HCP materials [48], resulting from the large BET surface areas (Figure 5A).

Compared to nitrogen physisorption, the benzene vaporsorption isotherms show a much more pronounced hysteresis over the whole relative pressure range. This effect can be explained by the strong interactions of the benzene molecules with the aromatic surface of the polymer via $\pi-\pi$ interactions. The rather strong adsorption at the inner surface is overlapped by pore filling due to a condensation-like effect. Thereby, swelling results in a reversible structural change. Interestingly, this swelling effect occurs only moderately up to a temperature of $298 \mathrm{~K}$. With further increasing temperature the swelling is much more pronounced with a significantly higher uptake at high relative pressure by condensation and a very pronounced hysteresis upon pressure-driven desorption (Figure 5B). A very
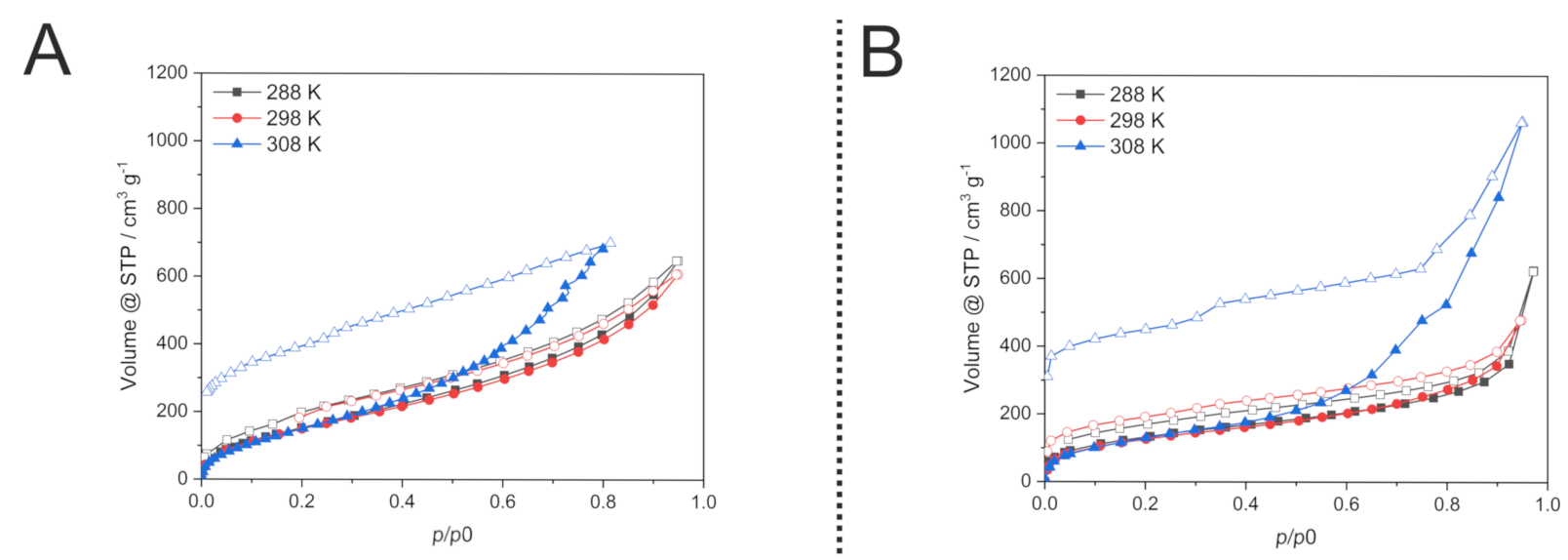

Figure 5: Physisorption isotherms of benzene vapour at different temperatures on HCP synthesised classically from solution (left, A) and mechanochemically (right, B). Filled symbols denote adsorption and open symbols desorption, respectively. 
similar behaviour is observed for both HCP samples LAG-HCP and Sol-HCP. However, the Sol-HCP shows a steeper slope of the adsorption isotherm due to the broader pore size distribution. In contrast, the LAG-HCP with the narrow PSD shows a more pronounced uptake at low relative pressures.

In addition, cyclohexane vapour was measured in the same temperature range (Figure 6A). Over the whole relative pressure region a lower uptake is observed compared to the benzene vapour. In a similar fashion, but slightly weaker pronounced, an adsorption-desorption-hysteresis is found at higher temperature $>298 \mathrm{~K}$ due to severe swelling. The difference in the uptake towards a favoured adsorption of benzene already points towards the potential for an adsorptive separation process of aromatic and aliphatic molecules with very similar physical properties.

To quantify the strength of adsorptive interactions from isotherms at various temperatures the isostatic heat of adsorption is calculated. Although in case of flexible materials with varying textural properties during adsorption and hence, a varying surface potential, this method should only be applied with great care and only used for a relative comparison of similar materials [49]. Absolute values calculated by this method are prone to great errors and should not be over-interpreted. Still, in our case the mechanochemically synthesised HCP shows an adsorption enthalpy at low loading of benzene of $70-80 \mathrm{~kJ} \mathrm{~mol}^{-1}$ that steadily decreases until it approximates the condensation enthalpy of pure benzene (Figure 6B). Interestingly, the heat of adsorption of cyclohexane is much lower with a maximum at low loading of approximately $50 \mathrm{~kJ} \mathrm{~mol}^{-1}$ and also decreasing until the condensation enthalpy is reached. Also, the condensation enthalpy of cyclohexane is reached at ca. $40 \%$ lower loading compared to benzene. This data points out the signifi- cantly favoured adsorption of benzene due to aromatic $\pi-\pi$ interaction in comparison to the significantly weaker bonding of aliphatic cyclohexane.

\section{Conclusion}

Summing up, we have demonstrated that mechanochemistry can be a suitable alternative in the synthesis of hyper-crosslinked polymers. By avoiding chlorinated solvents (typically 1,2dichloroethane), the synthesis of this promising material can be undertaken in a greener, faster, and cheaper fashion. The obtained materials show surface areas as high as $1720 \mathrm{~m}^{2} \mathrm{~g}^{-1}$ with a narrower pore size distribution compared to solvent-based analogues. It has been found that the addition of small amounts of liquid (LAG) is not only reducing the vessel pressure during the synthesis, but is also beneficial towards the textural properties of the material. In organic vaporsorption experiments the adsorption of benzene was favoured over cyclohexane by strong $\pi-\pi$ interaction with the aromatic framework. This has been proven by differences in uptake as well as by comparison of isosteric heat of adsorptions as direct indicator of the adsorption interactions.

\section{Experimental}

In our standard procedure HCP was synthesised through a mechanochemical reaction. This was accomplished by transferring 0.821 g 4,4'-bis(chloromethyl)-1,1'-biphenyl (SigmaAldrich) and $3.179 \mathrm{~g}$ (six equiv) of anhydrous iron(III) chloride (Sigma-Aldrich) as mediator into a $45 \mathrm{~mL}$ zirconium oxide milling vessel filled with 22 balls $(10 \mathrm{~mm}, 3.19 \pm 0.05 \mathrm{~g})$ of the same material. Consequently, the vessel was transferred into a P7 premium line (Fritsch $\mathrm{GmbH}$ ) and milled at $500 \mathrm{rpm}$ for 35 minutes. After the synthesis the resulting polymers were washed with $200 \mathrm{~mL}$ of water and $100 \mathrm{~mL}$ of ethanol and dried at $80{ }^{\circ} \mathrm{C}$ for 12 hours.
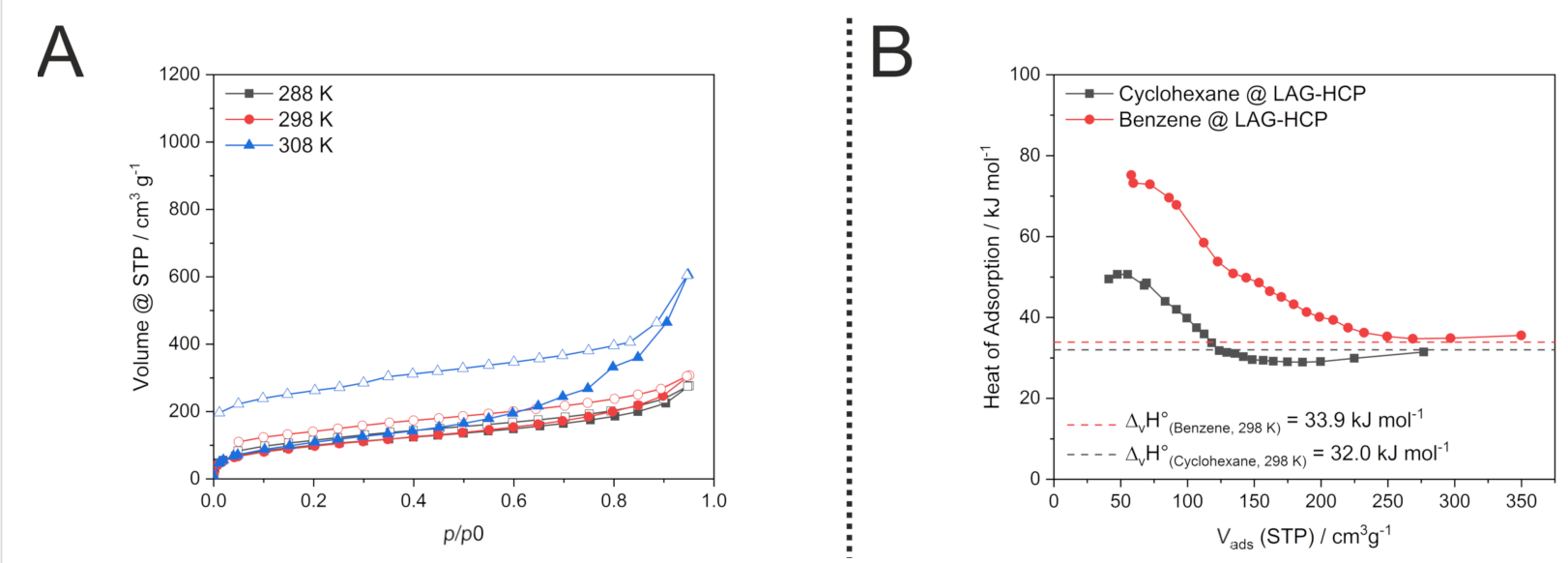

Figure 6: Physisorption isotherms of cyclohexane on mechanochemically synthesised HCP at temperatures 288,298 and $303 \mathrm{~K}$ (left) and isosteric heat of adsorption of benzene and cyclohexane on HCP synthesised mechanochemically (LAG-HCP) (right). 
For the LAG experiments the same quantities were used and $1 \mathrm{~mL}$ of the given liquid was added prior to the milling. The synthesis of the reference HCP from solution was carried out according to the procedure of Schute et al. [11]. Nitrogen physisorption measurements were performed at $77 \mathrm{~K}$ on an Autosorb-IQ-C-XR and Quadrasorb (Quantachrome Instruments). High purity gases were used for physisorption measurements $\left(\mathrm{N}_{2}: 99.999 \%\right)$. Specific surface areas $\left(\mathrm{SSA}_{\mathrm{BET}}\right)$ were calculated using the equation from Brunauer, Emmet and Teller (BET) in a relative pressure range that fits to the consistency criteria proposed by Rouquerol and Llewellyn. Pore size distributions were calculated using the quenched solid density functional theory (QSDFT) method for carbon (slit pores, equilibrium kernel) on the adsorption branch. Total pore volumes were determined from the adsorption branch at $p / p_{0}=0.95$. Prior to physisorption experiments, all samples were activated at $353 \mathrm{~K}$ for $24 \mathrm{~h}$ under vacuum. Ball mill syntheses were carried out in a Fritsch Pulverisette 7 premium line planetary ball mill. Infrared spectroscopy (IR) was carried out on a Bruker Vertex 70 with a Specac Golden Gate ATR unit. A resolution of $2 \mathrm{~cm}^{-1}$ was utilized and the resulting spectra were treated with ATR-correction by the OPUS 6.5 software. Powder X-ray diffraction (PXRD) patterns were collected in transmission geometry (MYTHEN 1K detector) with a STOE STADI P diffractometer operated at $40 \mathrm{kV}$ and $30 \mathrm{~mA}$ with a Ge monochromator using $\mathrm{Cu} \mathrm{K} \alpha_{1}$ radiation. Scanning electron microscopy (SEM/EDX) images were obtained using a Hitachi SU8020 SEM equipped with a secondary electron (SE) detector. Prior to the measurement the samples were prepared on an adhesive carbon pad and sputtered with gold to obtain the necessary electron conductivity. Thermogravimetric analysis (TGA) was performed on a Netzsch STA 409 PC/PG system using alumina crucibles under argon stream with the heating rate of $10 \mathrm{~K} \mathrm{~min}^{-1}$. The vapor sorption experiments were carried out using a Autosorb iQ from Quantachrome equipped with a vapor source and a heated manifold. Before evaporation the adsorptive solvent was dried using molecular sieves $4 \AA$ and degassed under vacuum.

\section{Supporting Information}

\section{Supporting Information File 1}

Additional information and figures.

[https://www.beilstein-journals.org/bjoc/content/

supplementary/1860-5397-15-112-S1.pdf]

\section{Acknowledgements}

We gratefully acknowledge the Federal Ministry of Education and Research (Bundesministerium für Bildung und Forschung, BMBF, award number 03SF0498) and the DECHEMA (MaxBuchner Forschungsstipendium) for financial support. The authors acknowledge Sebastian Ehrling for the SEM/EDX measurements. MR gratefully acknowledges funding by the German Research Foundation (Grant No. RO 4757/2-1).

\section{ORCID ${ }^{\circledR}$ iDs}

Sven Grätz - https://orcid.org/0000-0001-6026-097X

Marcus Rose - https://orcid.org/0000-0001-8196-1353

Lars Borchardt - https://orcid.org/0000-0002-8778-7816

\section{References}

1. Schüth, F.; Sing, K. S. W.; Weitkamp, J., Eds. Handbook of Porous Solids; Wiley-VCH Verlag GmbH: Weinheim, Germany, 2002. doi:10.1002/9783527618286

2. Schneemann, A.; Bon, V.; Schwedler, I.; Senkovska, I.; Kaskel, S.; Fischer, R. A. Chem. Soc. Rev. 2014, 43, 6062-6096. doi:10.1039/c4cs00101j

3. Ding, S.-Y.; Wang, W. Chem. Soc. Rev. 2013, 42, 548-568. doi:10.1039/c2cs35072f

4. Chaoui, N.; Trunk, M.; Dawson, R.; Schmidt, J.; Thomas, A. Chem. Soc. Rev. 2017, 46, 3302-3321. doi:10.1039/c7cs00071e

5. Martín, C. F.; Stöckel, E.; Clowes, R.; Adams, D. J.; Cooper, A. I.; Pis, J. J.; Rubiera, F.; Pevida, C. J. Mater. Chem. 2011, 21, 5475-5483. doi:10.1039/c0jm03534c

6. Li, C.; Liu, M.; Pschirer, N. G.; Baumgarten, M.; Müllen, K. Chem. Rev. 2010, 110, 6817-6855. doi:10.1021/cr100052z

7. Kuhn, P.; Antonietti, M.; Thomas, A. Angew. Chem., Int. Ed. 2008, 47, 3450-3453. doi:10.1002/anie.200705710

8. Pandey, P.; Katsoulidis, A. P.; Eryazici, I.; Wu, Y.; Kanatzidis, M. G.; Nguyen, S. T. Chem. Mater. 2010, 22, 4974-4979. doi:10.1021/cm101157w

9. Ritter, N.; Senkovska, I.; Kaskel, S.; Weber, J. Macromol. Rapid Commun. 2011, 32, 438-443. doi:10.1002/marc.201000714

10. Tan, L.; Tan, B. Chem. Soc. Rev. 2017, 46, 3322-3356. doi:10.1039/c6cs00851h

11. Schute, K.; Rose, M. ChemSusChem 2015, 8, 3419-3423. doi:10.1002/cssc.201500829

12. Rightmire, N. R.; Hanusa, T. P. Dalton Trans. 2016, 45, 2352-2362. doi:10.1039/c5dt03866a

13. Tan, D.; Loots, L.; Friščić, T. Chem. Commun. 2016, 52, 7760-7781. doi:10.1039/c6cc02015a

14. Xu, C.; De, S.; Balu, A. M.; Ojeda, M.; Luque, R. Chem. Commun. 2015, 51, 6698-6713. doi:10.1039/c4cc09876e

15. Bolm, C.; Hernández, J. G. Angew. Chem., Int. Ed. 2019, 58, 3285-3299. doi:10.1002/anie.201810902

16. Michalchuk, A. A. L.; Tumanov, I. A.; Boldyreva, E. V. CrystEngComm 2019, 21, 2174-2179. doi:10.1039/c8ce02109k

17. Tan, D.; García, F. Chem. Soc. Rev. 2019, 48, 2274-2292. doi:10.1039/c7cs00813a

18. Howard, J. L.; Cao, Q.; Browne, D. L. Chem. Sci. 2018, 9, 3080-3094. doi:10.1039/c7sc05371a

19. Andersen, J.; Mack, J. Green Chem. 2018, 20, 1435-1443. doi:10.1039/c7gc03797j

20. Do, J.-L.; Friščić, T. Synlett 2017, 28, 2066-2092. doi:10.1055/s-0036-1590854

21. Užarević, K.; Wang, T. C.; Moon, S.-Y.; Fidelli, A. M.; Hupp, J. T.; Farha, O. K.; Friščić, T. Chem. Commun. 2016, 52, 2133-2136. doi:10.1039/c5cc08972g 
22. Leistenschneider, D.; Jäckel, N.; Hippauf, F.; Presser, V.; Borchardt, L. Beilstein J. Org. Chem. 2017, 13, 1332-1341. doi:10.3762/bjoc.13.130

23. Schneidermann, C.; Jäckel, N.; Oswald, S.; Giebeler, L.; Presser, V.; Borchardt, L. ChemSusChem 2017, 10, 2416-2424. doi:10.1002/cssc.201700459

24.Zhang, E.; Hao, G.-P.; Casco, M. E.; Bon, V.; Grätz, S.; Borchardt, L. J. Mater. Chem. A 2018, 6, 859-865. doi:10.1039/c7ta10783h

25. Karak, S.; Kandambeth, S.; Biswal, B. P.; Sasmal, H. S.; Kumar, S.; Pachfule, P.; Banerjee, R. J. Am. Chem. Soc. 2017, 139, 1856-1862. doi:10.1021/jacs.6b08815

26. Karadeniz, B.; Howarth, A. J.; Stolar, T.; Islamoglu, T.; Dejanović, I.; Tireli, M.; Wasson, M. C.; Moon, S.-Y.; Farha, O. K.; Friščić, T.; Užarević, K. ACS Sustainable Chem. Eng. 2018, 6, 15841-15849. doi:10.1021/acssuschemeng.8b04458

27. Fidelli, A. M.; Karadeniz, B.; Howarth, A. J.; Huskić, I.; Germann, L. S.; Halasz, I.; Etter, M.; Moon, S.-Y.; Dinnebier, R. E.; Stilinović, V.; Farha, O. K.; Friščić, T.; Užarević, K. Chem. Commun. 2018, 54, 6999-7002. doi:10.1039/c8cc03189d

28. Chen, Y.; Wu, H.; Liu, Z.; Sun, X.; Xia, Q.; Li, Z. Ind. Eng. Chem. Res. 2018, 57, 703-709. doi:10.1021/acs.iecr.7b03712

29. Casco, M. E.; Badaczewski, F.; Grätz, S.; Tolosa, A.; Presser, V.; Smarsly, B. M.; Borchardt, L. Carbon 2018, 139, 325-333. doi:10.1016/j.carbon.2018.06.068

30. Ohn, N.; Shin, J.; Kim, S. S.; Kim, J. G. ChemSusChem 2017, 10, 3529-3533. doi:10.1002/cssc.201700873

31. Grätz, S.; Borchardt, L. RSC Adv. 2016, 6, 64799-64802. doi:10.1039/c6ra15677k

32. Ravnsbæk, J. B.; Swager, T. M. ACS Macro Lett. 2014, 3, 305-309. doi:10.1021/mz500098r

33. Malca, M. Y.; Ferko, P.-O.; Friščić, T.; Moores, A. Beilstein J. Org. Chem. 2017, 13, 1963-1968. doi:10.3762/bjoc.13.191

34. Ohn, N.; Kim, J. G. ACS Macro Lett. 2018, 7, 561-565. doi:10.1021/acsmacrolett.8b00171

35. Grätz, S.; Wolfrum, B.; Borchardt, L. Green Chem. 2017, 19, 2973-2979. doi:10.1039/c7gc00693d

36. Zhu, X.; Tian, C.; Jin, T.; Browning, K. L.; Sacci, R. L.; Veith, G. M.; Dai, S. ACS Macro Lett. 2017, 6, 1056-1059. doi:10.1021/acsmacrolett.7b00480

37. Troschke, E.; Grätz, S.; Lübken, T.; Borchardt, L. Angew. Chem. 2017, 129, 6963-6967. doi:10.1002/ange.201702303

38. Jamal, R.; Shao, W.; Xu, F.; Abdiryim, T. J. Mater. Res. 2013, 28, 832-839. doi:10.1557/jmr.2013.23

39. Biswal, B. P.; Chaudhari, H. D.; Banerjee, R.; Kharul, U. K. Chem. - Eur. J. 2016, 22, 4695-4699. doi:10.1002/chem.201504836

40. Zhu, X.; Hua, Y.; Tian, C.; Abney, C. W.; Zhang, P.; Jin, T.; Liu, G.; Browning, K. L.; Sacci, R. L.; Veith, G. M.; Zhou, H.-C.; Jin, W.; Dai, S. Angew. Chem., Int. Ed. 2018, 57, 2816-2821. doi:10.1002/anie.201710420

41. Posudievsky, O. Y.; Kozarenko, O. A. Polym. Chem. 2011, 2, 216-220. doi:10.1039/c0py00212g

42. Grätz, S.; Oltermann, M.; Troschke, E.; Paasch, S.; Krause, S.; Brunner, E.; Borchardt, L. J. Mater. Chem. A 2018, 6, 21901-21905. doi:10.1039/c8ta03684e

43. Rose, M.; Klein, N.; Senkovska, I.; Schrage, C.; Wollmann, P.; Böhlmann, W.; Böhringer, B.; Fichtner, S.; Kaskel, S. J. Mater. Chem. 2011, 21, 711-716. doi:10.1039/c0jm02998j

44. Grätz, S.; Beyer, D.; Tkachova, V.; Hellmann, S.; Berger, R.; Feng, X.; Borchardt, L. Chem. Commun. 2018, 54, 5307-5310. doi:10.1039/c8cc01993b
45. Andersen, J.; Mack, J. Angew. Chem., Int. Ed. 2018, 57, 13062-13065 doi:10.1002/anie.201805263

46. Detoni, C.; Gierlich, C. H.; Rose, M.; Palkovits, R. ACS Sustainable Chem. Eng. 2014, 2, 2407-2415. doi:10.1021/sc5004264

47. Schute, K.; Detoni, C.; Kann, A.; Jung, O.; Palkovits, R.; Rose, M. ACS Sustainable Chem. Eng. 2016, 4, 5921-5928. doi:10.1021/acssuschemeng.6b00096

48. Wang, G.; Dou, B.; Wang, J.; Wang, W.; Hao, Z. RSC Adv. 2013, 3, 20523-20531. doi:10.1039/c3ra41450g

49. de Lange, M. F.; Verouden, K. J. F. M.; Vlugt, T. J. H.; Gascon, J.; Kapteijn, F. Chem. Rev. 2015, 115, 12205-12250. doi:10.1021/acs.chemrev.5b00059

\section{License and Terms}

This is an Open Access article under the terms of the Creative Commons Attribution License (http://creativecommons.org/licenses/by/4.0). Please note that the reuse, redistribution and reproduction in particular requires that the authors and source are credited.

The license is subject to the Beilstein Journal of Organic Chemistry terms and conditions: (https://www.beilstein-journals.org/bjoc)

The definitive version of this article is the electronic one which can be found at: doi:10.3762/bjoc. 15.112 